\title{
GENERATING GRAPHS IN VIRTUAL REALITY
}

\author{
Simon So \\ The Education University of Hong Kong \\ 10 Lo Ping Road, Tai Po, N.T., Hong Kong
}

\begin{abstract}
Recent advances in Head-mounted Display (HMD) for Virtual Reality have been phenomenal. The quality of the latest HMDs such as Oculus Rift, HTC Vive and Sony PlayStation VR is high. These highly immersive devices create educational opportunities as well as challenges. Specifically, we can create or view graphs in a virtual environment. This innovative technology of generating graphs opens up a new horizon. We can plot 3D graphs so that the user can view the graphs from any angles and examine the graphs virtually. Furthermore, dynamic data can be summarized into diagrams and graphs. If such data are fed into the virtual system, we can view the change of the graphs virtually. In this paper, we will demonstrate the presentation of graphs using HMDs in Virtual Reality. We will illustrate hand-drawings and system-generated graphs in virtual spaces. Students' views on creating graphs in Virtual Reality will be examined. An overview of VR devices and software to produce graphs will also be discussed.
\end{abstract}

\section{KEYWORDS}

Virtual Reality, Head-mounted Display, Graphing, Virtual Data Visualization

\section{INTRODUCTION}

With the proliferation of consumer-level head-mounted displays and motion tracking devices, virtual reality experience has been phenomenal in recent years. Recently, the releases of Oculus Rift (2019), HTC Vive (2019) and Sony PlayStation VR (2019) have changed the consumer market of virtual reality significantly. We can now play highly immersive VR games with these devices. To educators, VR devices are becoming more affordable and accessible. VR applications are being released constantly. The education sector, the entertainment industry, the business sector and many other fields can be benefited from many of these applications.

In this paper, we will explore how VR can be used in creating virtual 3D graphs. We are interested to generating graphs virtually by either sketching or generating graphs in virtual space. Sketching graphs can promote learning (Fiorella \& Mayer, 2016) and this is an important pedagogical approach for teachers. We will explore hand-drawing graphs in virtual space. This experience is unique and the approach may be useful for teachers.

Graphs and figures can be generated by machines. For example, a virtual reality system may take instructions from upstream systems to generate virtual models of molecular figures in Organic Chemistry (Stull, Barrett \& Hegarty, 2012). Their findings suggest a benefit of using virtual models over concrete models. In this paper, we are more interested on graphs; system-generated graphs. These graphs are being generated in a virtual space by static data files or pre-processed data from dynamic sources. For example, stock market information can be accessed through Virtual Reality (Sá \& Dähne, 2016). Many immersive and intuitive ways to interact with graphs can be found. This opens up a range of innovations and challenges to draw graphs.

In Section 2, we will discuss hand-drawing and system-generated graphs. Some of the possibilities are outlined in this section. The students' views on using VR for educational purposes and creating virtual reality graphs are revealed in Section 3. Section 4 will provide a summary discussion. 


\section{GRAPHING IN VIRTUAL REALITY}

\subsection{Hand-Drawing Graphs}

Painting in 3D space with virtual reality has been made popular by Google Tilt Brush (2019). Tilt Brush allows the creator to completely immerse in virtual reality for 3D painting using paddles like HTC Vive's controllers or Oculus Touch controllers with their respective head-mounted displays. The creative potential of this technology is not restricted to artists, cartoonist, painters, designers, illustrators and other creators (TIME, 2019). Educators can make use of this tool for drawing, painting and sculpting. The tool allows educators to extend learning virtually. In our case, drawing graphs in virtual space are both creative and fascinating. Students can learn from graphs created by teachers in 3D space. They can walk $360^{\circ}$ around the graphs. Viewing from different orientations can provide different perspectives to learners. For example, if 3D vectors were drawn on a 3D graph, students can observe the vectors' projection on a $2 \mathrm{D}$ plane by walking to the perpendicular position of a principle axis. Figure 1 illustrates an example and the vectors' projection is shown in Figure 2. Furthermore, sketching graphs in a virtual space are both fun and challenging.

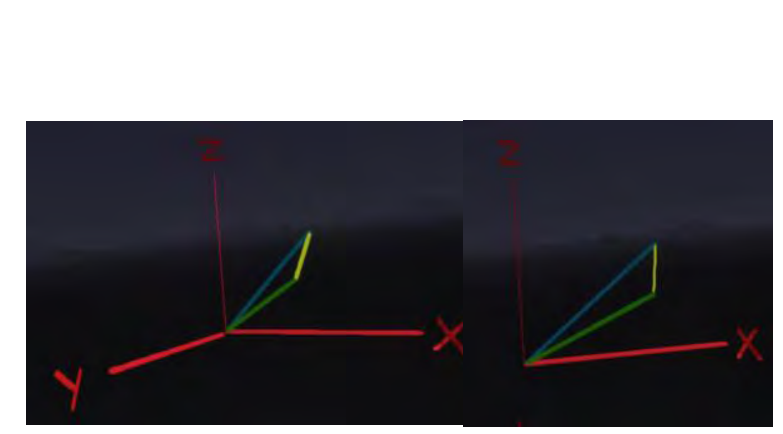

Figure 1. 3D vectors sketched on a virtual space

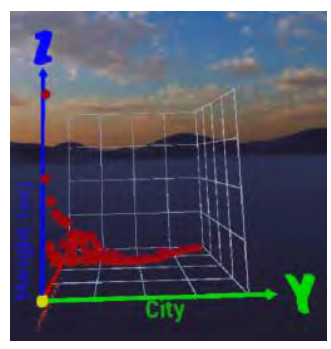

Figure 4. The plot of the tallest buildings in the world

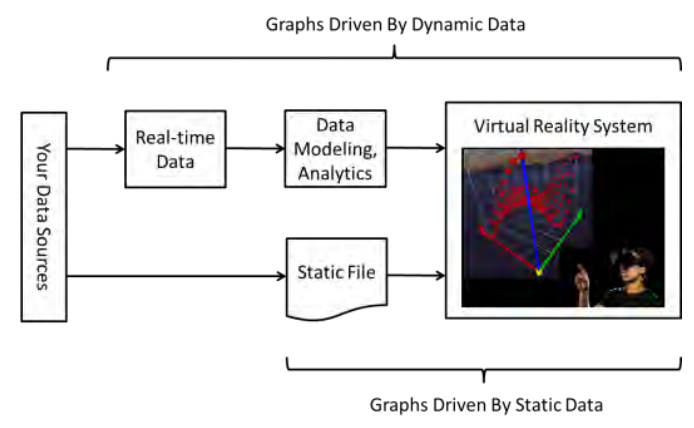

Figure 3. The concept of static and dynamic data driven graphs

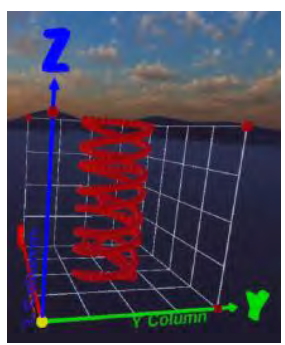

Figure 6. The plot of a double helix

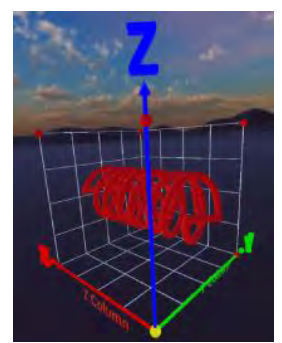

Figure 7. Swap the axes to see the helix differently

\subsection{System-generated Graphs}

Generating graphs in virtual reality can be carried out statically or dynamically. Data can be read from static files to generate graphs in a virtual space. Alternatively, data can be fed into the virtual reality system from dynamic sources (Sá \& Dähne, 2016). Figure 3 illustrates the concept. Depending on the sophistication of the underlying software, the viewer can decide the required types of graphs, manipulate the presentation, examine the individual data points, and view the graphs from different positions (DatavizVR, 2018; Virtualitics, 2019; LookVR, 2019). The virtual reality system can be programmed generically to visualize standardized data or specifically coded to handle incoming data and present the specific graphs interactively.

This approach offers educators a whole new manner for students to learn graphs. Students can interact with graphs driven by the data in a virtual space. For examples, students can select any data point in the 
graphs to examine the values. Figure 4 illustrates the examination of a data point by a student on the data set of the tallest buildings in the world in the 3D graph using the paddles in DatavizVR (2018). In this VR system, users can configure the $\mathrm{X}, \mathrm{Y}, \mathrm{Z}$-coordinates for 3D graphs. They can point and choose the data point to examine and view the data from all angles as shown in Figure 5. The examination of a double helix is shown in Figure 6 and 7. The color and size of each data point can be used to represent the fourth and fifth dimensions.

Dynamic data can be pre-processed and fed into a virtual reality system. To gain insights from the dynamic data sets, the data can be transformed by certain data modeling techniques or certain analytic tools as illustrated in Figure 3. These tools process and provide visualization and interaction of dynamic data in virtual space (Nagel, Granum, \& Musaeus, 2001; Olshannikova, et. al., 2015). Figure 8 shows the processed data of Olympic medalists from LookVR (2019). Artificial intelligence and machine learning are some of the common processing tools as shown in Figure 9 from Virtualitics (2019). Data from finance, healthcare, transportation, security and many other disciplines can be huge. These data are commonly referred as big data. If these big data are processed and fed into the virtual reality system (Moran, et. al., 2015; Marks, Estevez, \& Connor, 2014; Donalek, et. al., 2014), the viewers can immerse themselves in the processed data and inspect the dynamic nature of the data in a more natural and intuitive way. This revolutionizes the visualization of big data.

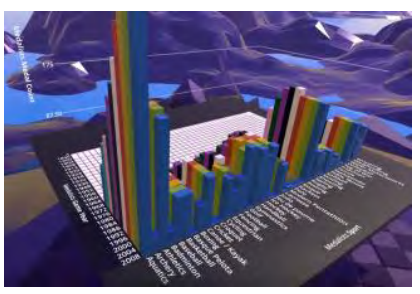

Figure 8. 3D bar charts of Olympic medalists

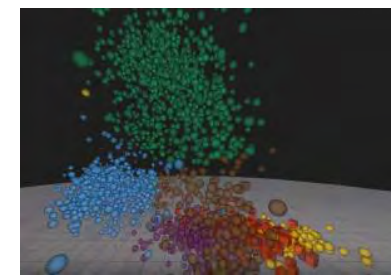

Figure 9. Visualizing data after processing techniques in $\mathrm{AI}$ and machine learning

\section{STUDENTS' VIEWS ON CREATING GRAPHS IN VIRTUAL REALITY}

Three students were asked to explore the applications of sketching and interacting with graphs in virtual reality. The lab has HTC Vive, Sony PlayStation VR, Oculus Rift and a number of handheld VR devices. The experiment was conducted mainly with HTC Vive. The other devices such as Sony PlayStation VR are mainly for VR games. The students worked with some of the above software. Interviews were conducted afterward. The following responses come from one of the students:

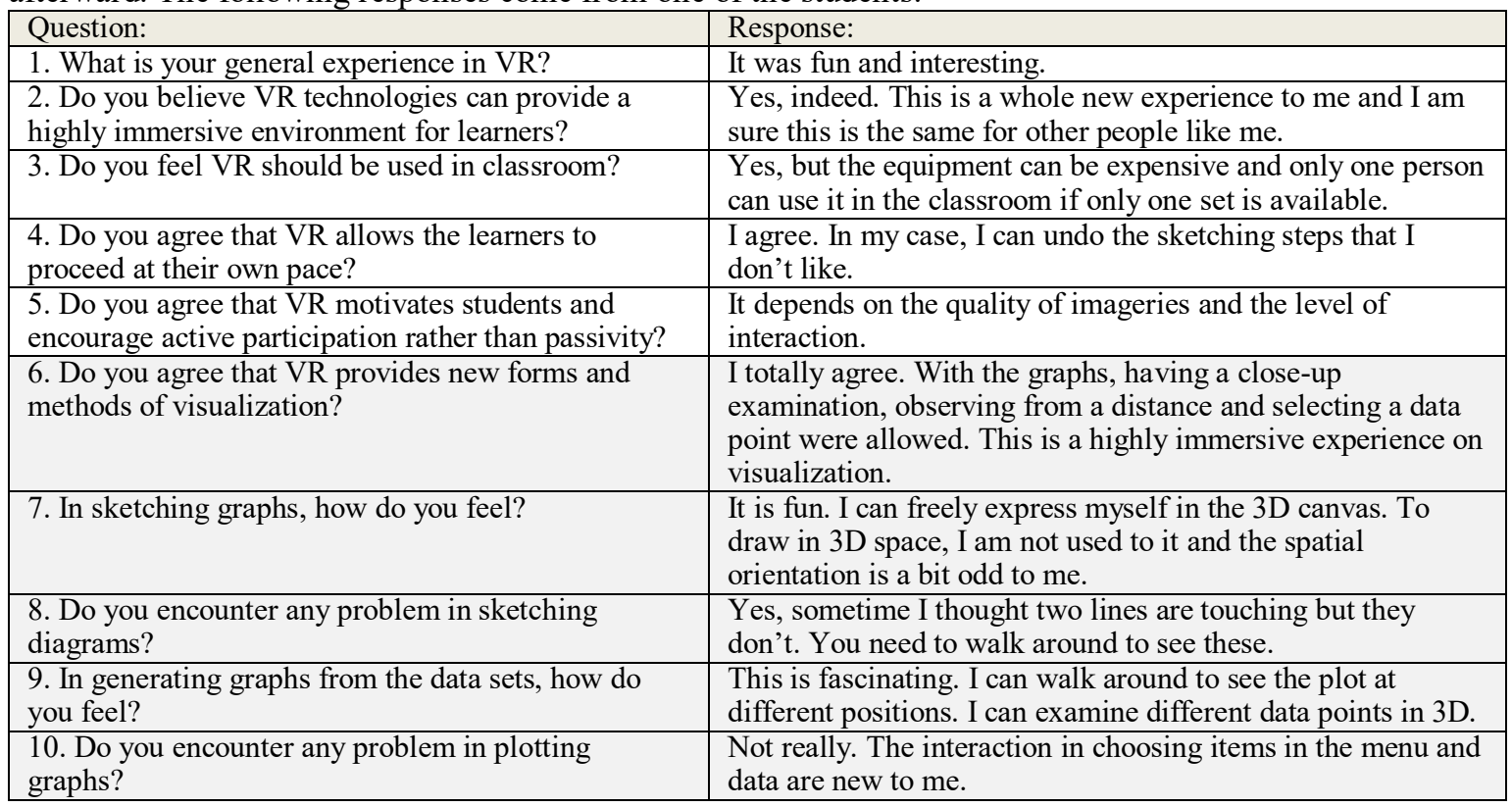




\section{DISCUSSION}

Sketching in Virtual Reality has never been so exciting before Google Tilt Brush (2019). The tool is not only good for artists (Tilt Brush AiR, 2019). You can also import objects into the virtual space which is a great feature for designers. The rich features in Tilt Brush allow educators to extend their teaching to Virtual Reality. In this experiment, we explored the use of this tool to sketch or draw graphs. Inside the virtual space, the users can freely express themselves. The analogy would be that students can use pencils or drawing tools to create graphs onto physical papers. They found some possibilities that the physical world does not have or cannot be easily achieved. The sketching process is intuitive. This is certainly an educational tool for sketching graphs in a virtual environment.

In system-generated graphs, we are interested in generating graphs from static and dynamic data. In the experiment, users can manipulate a range of features and examine data from any angle in the virtual space. The only limitation is from the software itself. Although these applications are still new to the market, we expect more universal applications will be launched in the coming years so that we can connect a range of structured and semi-structured data to the virtual systems. Visualization of small or big data can be more efficient, immersive and intuitive.

In Virtual Reality, it is possible to allow users from different locations to "teleport" to the virtual environment and work or learn collaboratively as a group. Each user is a fully featured avatar in the virtual environment. This feature can be useful and interesting for many tasks in education such as generating 3D graphs and in business. With the ever increasing power of hardware, the feature is around the corner.

Overall, students like the experience in Virtual Reality. They felt VR technologies can be useful for teaching and learning. They enjoy making graphs with the tools. They could proceed at their own pace.

\section{REFERENCES}

DatavizVR (2018). Dataviz VR. Retrieved on 15 April 2018, from http://www.datavizvr.com/

Donalek, C., Djorgovski, S. G., Cioc, A., Wang, A., Zhang, J., Lawler, E., \& Davidoff, S. (2014). Immersive and collaborative data visualization using virtual reality platforms. 2014 IEEE International Conference on Big Data. pp. 609-614. IEEE Press (2014, October)

Fiorella, L., \& Mayer, R. E. (2016). Effects of observing the instructor draw diagrams on learning from multimedia messages. Journal of Educational Psychology, 108(4), 528.

Google Tilt Brush (2019). Google Tilt Brush. Retrieved on 30 April 2019, from http://www.tiltbrush.com/

HTC Vive (2019). HTC Vive. Retrieved on 30 April 2019, from http://www.vive.com/

LookVR (2019). LookVR. Retrieved on 30 April 2019, from http://looker.com/vr/

Marks, S., Estevez, J., \& Connor, A. (2014). Towards the Holodeck: fully immersive virtual reality visualisation of scientific and engineering data. Proceedings of the 29th International Conference on Image and Vision Computing. New Zealand. ACM. pp. 42-47

Moran, A., Gadepally, V., Hubbell, M., \& Kepner, J. (2015). Improving Big Data visual analytics with interactive virtual reality. High Performance Extreme Computing Conference (HPEC), pp. 1-6. IEEE. (2015, September)

Nagel, H. R., Granum, E., \& Musaeus, P. (2001). Methods for visual mining of data in virtual reality. Proceedings of the International Workshop on Visual Data Mining, pp. 13--27 (2001, September)

Oculus Rift (2019). Oculus Rift S. Retrieved on 30 April 2019, from http://www.oculus.com/rift

Olshannikova, E., Ometov, A., Koucheryavy, Y., \& Olsson, T. (2015). Visualizing big data with augmented and virtual reality: challenges and research agenda. Journal of Big Data, 2:22.

Sá, V. J., \& Dähne, P. (2016). Accessing Financial Data through Virtual Reality. Atas da Conferência da Associação Portuguesa de Sistemas de Informação. Vol. 3. No. 3.

Sony PlayStation VR (2019). Sony PS4 VR. Retrieved on 30 April 2019, from https://www.playstation.com/

Tilt Brush AiR (2019). Tilt Brush Artist in Residence. Retrieved on 30 April 2019, from http://www.tiltbrush.com/air/

Stull, A. S., Barrett, T. J., \& Hegarty, M. (2012). Design of a virtual reality system for the study of diagram use in organic chemistry. Proceedings of the workshop on technology-enhanced diagrams research. pp.33-41.

TIME (2019). Virtual reality for artists. Retrieved on 30 April 2019, from http://time.com/vr-is-for-artists/

Virtualitics (2019). Virtualitics. Retrieved on 30 April 2019, from http://www.virtualitics.com/

HTC Vive (2019). HTC Vive. Retrieved on 30 April 2019, from http://www.vive.com/ 